\title{
Dynamics of vibratory partitioned feeders for the uranium ore drawing and feeding
}

\author{
Vitalii Dyrda ${ }^{1, *}$, Anatolii Kobets ${ }^{2}$, Viktor Pukhalskyi ${ }^{3}$, Yurii Kozub ${ }^{4}$, and Oleksandr \\ Chernii $^{1}$ \\ ${ }^{1}$ Institute of Geotechnical Mechanics named by N. Poljakov of National Academy of Sciences of \\ Ukraine, 49005, Dnipro, Simferopolska Str., 2a, Ukraine \\ ${ }^{2}$ Dnipro State Agrarian and Economic University, 49600, Dnipro, Serhiia Yefremova Str., 25, \\ Ukraine \\ ${ }^{3}$ Estern Mining and Processing Plant, 52210, Zhovti Vody, Horkoho Str., 2, Ukraine \\ ${ }^{4}$ Luhansk Taras Shevchenko National University, 92700, Starobilsk, Hoholia Sq., 2, Ukraine
}

\begin{abstract}
In this paper, the authors consider dynamics of partitioned vibratory feeders with rubber elastic couplings. Use of vibratory feeders (including partitioned ones) and schemes for the ore vibrating drawing, feeding and loading in underground conditions allowed to mechanize one of the most time-consuming and dangerous processes of uranium ore mining and to solve the following problems: to improve effectiveness of the lumpy rock mass drawing and loading by 2.5-3 times; to increase intensity of mining operations and reduce life of the excavation blocks and horizons; to reduce labor intensity and cut cost of the process of ore drawing and loading; to improve uniformity of the rock-mass outflowing from the discharge opening and to reduce by 3-5 times frequency of the large-lump material freezing (use of partitioned feeders reduces number of freezes by 25-60 times); to increase size of commercial lump panels; practically to exclude traumas during the process of ore drawing and loading (usually, it presents $30-60 \%$ of all traumas caused by the underground operations); to mechanize process of ore drawing and loading, and to create conditions for implementation of low-waste cyclicand-continuous mining technology.
\end{abstract}

\section{Introduction}

Rate of the world economy growth is inextricably associated with development of energy industry. According to the International Energy Agency, world energy consumption will soon increase by one third; its deficit will be covered, among other things, by intensive development of nuclear power engineering. Today, nuclear-power industry is the only stably developing industry, which successfully competes with traditional sources of energy, such as oil, gas, coal, etc. Today, there are 15 nuclear power-generating units in Ukraine with a total capacity of more than 15 million $\mathrm{kW}$ presenting almost half of the country's total electricity. Ukraine is provided with sufficient reserves of raw materials and is among the top ten world producers of uranium. Ukraine began produce uranium ore in 1946 in the

\footnotetext{
*Corresponding author: vita.igtm@gmail.com
} 
Zhovtorichenske and Pershotravenske deposits; since 1951, all works were performed by the ore-mining complex \#9 (now, it is the state enterprise "Eastern Mining and Processing Plant" in the city of Zhovti Vody). The state company "Estern Mining and Processing Plant" is the only enterprise, which extracts and processes uranium ores and produces natural uranium concentrate in full harmony with the world standards. Uranium ore is extracted by underground method; ore is drawn and fed from the panels by heavy mining vibratory feeders.

Today, underground method is used in all low-grade deposits and for mining at great depths in difficult mining, geological and technical conditions. At the same time, these complications decrease rate of development of underground ore mining and require significant improvement for mining equipment and technologies.

One of the most important technological processes in ore-deposit underground mining is drawing of broken rock from the working excavation zone and loading it into transport facilities. This process's share in total labor costs is up to $60 \%$ and is associated with high level of injury of miners (up to 30-60\% of total underground mining operations).

The authors' scientific studies and their experience in designing and commissioning heavy vibratory-type machines show that the problem of highly effective and safety ore drawing and loading from the extraction blocks can be successfully solved by using vibratory feeders and complexes, which intensively effect the drawn material, reduce energy consumption and number of material freezes and ensure intensification and concentration of mining operations. Other technologies are also available, however, the vibratory drawing is still relevant today [1].

Vibratory drawing fully meets all modern challenges as it allows operating with rockmass lumps with size of up to 1.2-1.4 m, ensuring technical productivity of $1500-2000 \mathrm{t} / \mathrm{hr}$ and producing not less than 1.2-1.5 million tons.

The overall decrease of mining operation rate and complications associated with deep horizons required intensification and concentration of mining operations in blocks and deposits, use of highly effective development systems with mass ore breakage by blasthole explosive charges, and transition to cyclic-and-continuous and continuous underground mining technologies. At the same time, the most critical constrain in this case was freezing of lumpy rocks.

In order to eliminate this negative effect and to intensify the process as a whole, an original scheme was studied and designed for the vibratory partitioned feeders, which allows to expand zone of vibration impact on the drawn material and, instead of discharge opening, to use discharge slot, which is significantly greater than maximum size of the drawn lumpy material. A new method of sectioning is used in design of small-sized vibratory feeders PVM-1,0/2,3, vibratory partitioned feeders PVS-1,4/7,0, and in vibratory partitioned mining complexes KVGS-1 (Fig. 1, Fig. 2).

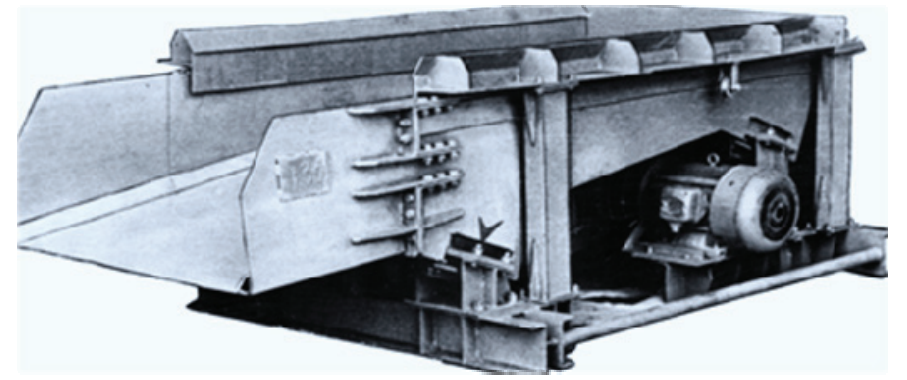

Fig. 1. Vibratory feeder of the VPR-4m type with the rubber elastic suspesion (BRM type). 


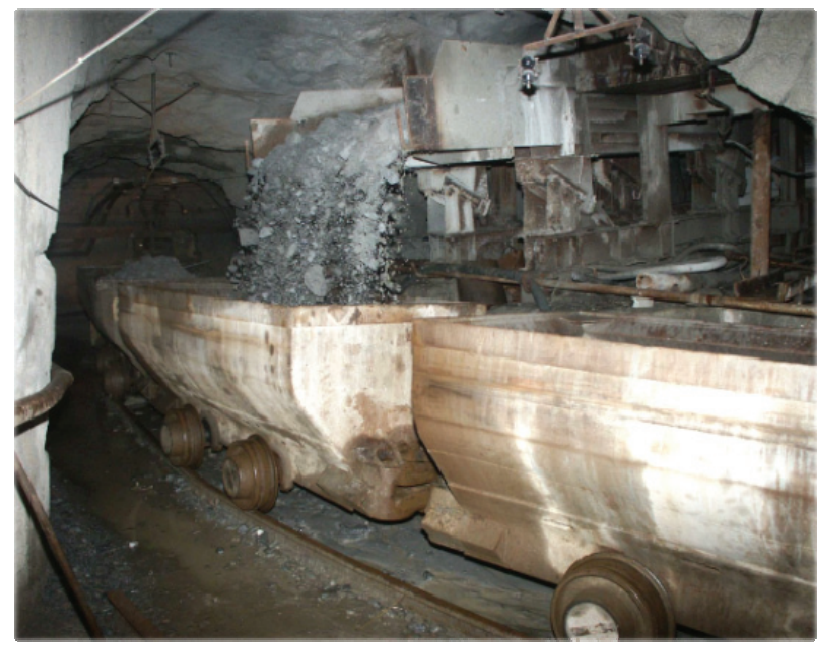

Fig. 2. Vibratory feeder of the VPR- $4 m$ type for the ore drawing and feeding from the panel to the wagons.

Use of vibratory partitioned feeders and complexes instead of conventional ones allowed to reduce volume of tunneling operations, cut ore loss in the bottoms of the blocks and improve productivity and reliability of the process due to the much less number of freezes (by 25-60 times) with no additional expenditure of energy.

Uranium ore drawing with the help of vibratory feeders allows to expand zone of impact on the drawn rock mass, increase active cross-section of the stream and reduce risk of arch formation and, accordingly, number of freezes. Intensity of vibratory feeder action on the material in extraction zone is determined by the depth of penetration, which is about $0.4 \mathrm{~m}$ and can vary depending on operating conditions and method of breaking.

In order to make penetration deeper, it is necessary to increase significantly length of vibratory feeder, drive power, metal consumption and costs of assembling and disassembling. Besides, it should be mentioned that with increasing depth, static loads on the feeder and its elastic system also increase and can reach 50 tons or more [3].

Problems of uranium ore drawing and feeding are described in [2-4], where designs of vibratory feeders and some specific features of their operation are considered. Though, problem of partitioned feeder dynamics is still unsolved.

Objective of the work: to develop method for calculating dynamic characteristics of partitioned vibratory feeders and to determine dependence of intrinsic vibration frequencies on the stiffness of elastic couplings in the drive element and drive section, length of platform and ratio of vibrating rock mass in the section.

\section{Method}

\subsection{Partitioned vibratory feeders}

Structurally, they are simple: drive section is made on the basis of a well-known feeder and is equipped with a platform buried under a straight column of broken rock mass, which periodically receives oscillatory energy from the drive section under condition that it is released from the mass of the drawn material. Platform of partitioned feeder is turned on automatically and expands zone of vibration action on the column of broken rock mass, hence, eliminating processes of arch formation. 
In Figure 3, general dynamic scheme of partitioned feeder is shown. Front drive section 1 (it is based on, for example, VPR-4m) forms a transport stream and is equipped with own independent drive 2. Depending on use environment, drive section 1 can be deepened to a certain depth under the column of broken rock mass. The second section 3 (hereinafter the platform) is located directly in the broken-down rock mass and does not have any drive. Platform 3 is turned on by restoring forces of elastic supports 4 in the drive section 1 , oscillating work member of which engages with the platform through the elastic drive element 5. At normal load, this drive section subsides on the elastic supports 4, disengages with the platform 3 and forms, between the platform and drive element $C_{1}$, a gap $\delta$, whose size is greater than or equal to the magnitude of oscillation amplitude of the drive section.

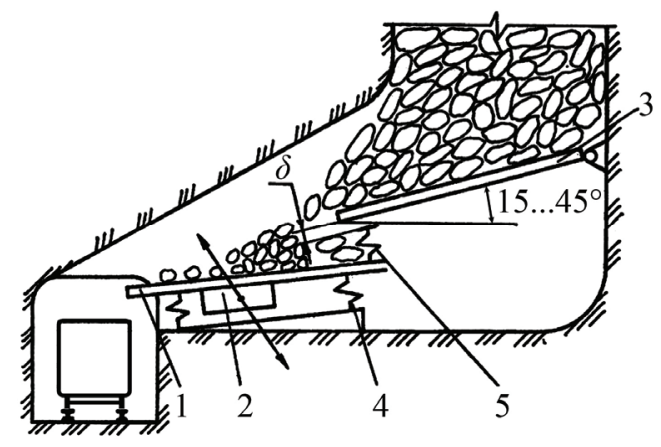

Fig. 3. Dynamic scheme of vibratory partitioned feeder: 1 - drive section; 2 - vibration exciter; 3 platform; 4 - elastic supports of the drive section; 5 - elastic drive element $C_{1} ; \delta$ - gap.

Rate of the drive elastic element compression and, consequently, rate of vibration transfer to the platform is inversely proportional to the amount of material on the work member in the drive section.

Certain differences in the kinematics of partitioned feeders, especially in kinematics and execution of their platform, predetermine a large variety of schemes for the ore drawing and loading in underground conditions, their dependence on the mining systems applied, thickness of ore bodies, needed completeness of excavation and geological and technical conditions of mining. Due to this, three types of partitioned feeders are proposed. Each of these types is characterized by method of platform supporting and type of the platform oscillations, and on the basis of these characteristics, strength and rigidity of the platform, its load-carrying capacity are determined. Depending on productivity and load-carrying capacity, partitioned feeders are conventionally divided into three types: light, medium and heavy.

Light-type feeders are designed for drawing and loading rock mass with size of up to $0.4 \mathrm{~m}$ when mining thin and vein deposits with ore shrinkage. Length of the platform is between $1 \mathrm{~m}$ to $2 \mathrm{~m}$. The platform is a plate, whose front end is rest on oscillating work member of the driving section with the help of elastic element of the drive, and rear end of the platform is connected by hinge to the wall of tunnel or bunker. Angle of the platform inclination can be between $15^{\circ}$ and $45^{\circ}$ depending on specific location of the feeder. During operation, the feeder platform makes two types of oscillations: bending and angular relative to the hinge.

Medium-type feeders are designed for drawing ore directly from the box holes in the excavation blocks. These feeders should withstand significant pressure caused by column of broken-down rock mass and dynamic loads caused by blasting operations, which are needed for forming cutting slot, eliminating material freezing and crushing oversize rocks. Platform of such feeders is a plate with length of $2-4 \mathrm{~m}$ of the same or variable stiffness, 
and it should be rested directly on the ore bench or longitudinal terrace at an angle of $20^{\circ}-40^{\circ}$.

Medium-type feeders are used for mining thick and very thick ore deposits when oversize lumps presents more than $5 \%$. Principle of the platform operation assumes that broken rock mass moves on its inclined surface under the action of gravitational forces due to reduced effective coefficients of friction and adhesion of ore to the platform surface under the action of vibration transferred to the platform from the oscillating work member through the elastic element. Platform of medium-type partitioned feeder produces bending oscillations.

Heavy-type feeders are designed for operating in the conditions of blockage when ore can be crushed by the borehole explosive charges, for example, at lateral drawing of ore. In this case, mining operations require that the feeder should move together with front of oreface operations and, hence, at multiple explosions of borehole charges. Length of the platform can be between $2 \mathrm{~m}$ and $4 \mathrm{~m}$ depending on step of the ore-face operation. In order to ensure more reliable drawing and loading operations, platform is rested on elastic couplings. Angle of the platform installation is $5^{\circ}-15^{\circ}$ to the horizon.

\subsection{Dynamics of partitioned feeders}

In terms of dynamics, the considered schemes of three types of partitioned feeders are multi-mass systems oscillating under the action of harmonic driving forces.

Calculations are based on the following assumptions: work member moves parallel to the plane; work member and platform are rigid bodies; dissipative forces are not taken into account; only vertical components of the oscillation amplitude are considered.

Let's consider light-type feeders.

Let's assume that $X$ is direction of work member oscillation, $\varphi$ is angular oscillations of the platform, $w$ is deflection of the platform.

The expressions of the kinetic and potential energies in the selected generalized coordinates for the light-type feeders have the form:

$$
\begin{aligned}
E_{k}= & \frac{m_{1} \dot{X}^{2}}{2}+\frac{J \dot{\varphi}^{2}}{2}+\frac{\gamma_{0} h_{0}}{2} \int_{0}^{l} \int_{0}^{b}\left(\frac{\partial w}{\partial t}\right)^{2} d x d y \\
E_{p}= & \frac{C_{1} X^{2}}{2}+\frac{C_{n}(X-w-\varphi l)^{2}}{2}+ \\
& +\frac{D^{l}}{2} \int_{0}^{l} \int_{0}^{b}\left\{\left(\frac{\partial^{2} w}{\partial x^{2}}+\frac{\partial^{2} w}{\partial y^{2}}\right)^{2}-2(1-\mu)\left[\frac{\partial^{2} w}{\partial x^{2}} \frac{\partial^{2} w}{\partial y^{2}}-\left(\frac{\partial^{2} w}{\partial x \partial y}\right)^{2}\right]\right\} d x d y,
\end{aligned}
$$

In deriving the differential equations of motion of the considered dynamic system, we use the Ostrogradsky-Hamilton principle, which for conservative systems has the form:

$$
S=\int_{t_{1}}^{t_{2}} L d t .
$$

where $L$ is the Lagrange function, $L=E_{k}-E_{p}$.

Then, composing on the basis of (1) the functional (2) and applying the Euler method, we obtain a system of differential equations describing the motion of a lightweight partitioned feeder 


$$
\left\{\begin{array}{l}
m_{1} \ddot{X}+\left(C_{1}+C_{n}\right) X-C_{n} \varphi l-C_{n} w(x, y, t)=P \sin \omega t \\
J \ddot{\varphi}+C_{n} \varphi l^{2}-C_{n} l X+C_{n} l w(x, y, t)=0 \\
D \Delta w(x, y, t)+m_{s} \frac{\partial^{2} w(x, y, t)}{\partial t^{2}}+C_{n} w(x, y, t)+C_{n} \varphi l-C_{n} X=0 .
\end{array}\right.
$$

In equations (1), (3) $J=m_{s} l^{2} / 3$ - moment of inertia of the platform relative to the hinge; $m_{s}$ is oscillating mass of the platform; $l, b$ is length and width of the platform; $\gamma_{0}, h_{0}$ is the density and thickness of the material of the platform; $D=\frac{E d^{3}}{12\left(1-\mu^{2}\right)}$ is cylindrical stiffness of the platform; $m_{1}$ is mass of the work member in the drive section; $C_{1}$ is stiffness of elastic supports in the drive section; $C_{n}$ is stiffness of elastic supports of the platform; $P=m g$ is load on the platform; $E, \mu$ is modulus of elasticity and Poisson's ratio for the platform material; $\Delta=\frac{\partial^{2}}{\partial x^{2}}+\frac{\partial^{2}}{\partial y^{2}}$ is the Laplace differential operator; $\omega$ is frequency of oscillations.

Forced oscillations of a partitioned feeder are determined from the solution of the inhomogeneous system of equations (3).

Solving of the frequency equations for all 3 types of feeders makes it possible to determine dependence of intrinsic frequency $f$ of the partitioned feeder on the stiffness of drive elastic element $C_{1}$, stiffness of elastic couplings of the drive section $C_{n}$, length of the platform $l$, and ratio of oscillating mass $m_{1} / m_{s}$ in the sections (Fig. 4).

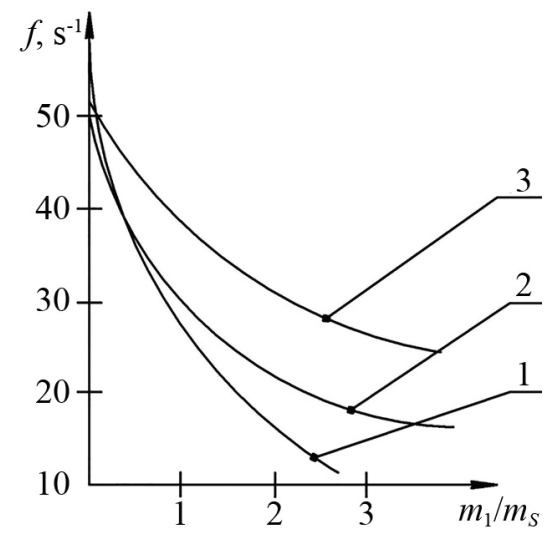

Fig. 4. Dependence between the partitioned feeder's intrinsic frequency $f$ and ratio of oscillating mass in sections $m_{1} / m_{s}: 1$ - light type; 2 - medium type; 3 - heavy type.

Rubber elements of elastic suspension in the platform and drive section were calculated by the method described in [5].

\section{Conclusions}

1. A new method was developed for calculating dynamic characteristics for the partitioned vibratory feeder and determining dependence of intrinsic oscillation frequency on parameters of the drive section, platform length and ratio of oscillating masses.

2. Vibratory machines and complexes created by the team of scientists and designers successfully operate in the mining enterprises of Ukrainian uranium industry, including 
PJSC "Northern Mining and Processing Plant” (Kryvyi Rih), PJSC “ArcelorMittal Kryvyi Rih", Krivyy Rih Iron Ore Plant, production association "Uralzoloto", Poltava OreDressing and Processing Enterprise, mining enterprises of uranium industry in the Russian Federation, Kazakhstan, Kyrgyzstan and Uzbekistan. Volume of the rock-mass vibratory drawing in the state enterprise "Estern Mining and Processing Plant" is about $100 \%$. Current annual manufacture is 20-25 vibratory feeders for different purposes.

\section{References}

1. Puhalskiy, V.N., Sinchuk, V.V., Basarab, N.N. (2017). Production and processing of uranium ore in Ukraine, Geo-Technical Mechanics, (133), 27-33

2. Poturaev, V.N., Dyrda, V.I. Poddubnyy, I.K. Avdeev, O.K., Gordienko, N.A., Koval, A.V., Finogeev, V.I., Lisitsa, N.I., Dudchenko, A.Kh. (1989). Vibrodostavochnyye kompleksy $v$ tekhnologiyakh razrabotki rudnykh mestorozhdeniy. Kyiv: Naukova Dumka

3. Kornev, G.N. (1988). Povyshenie effektivnosti vibratcionnogo vypuska rudy iz blokov na osnove izucheniia mehanizma protcessa. Leningrad: Nauka

4. Poddubniy, I.K. (1982). Industrial studies of operating modes and structural elements of vibrating mining feeders, Metallurgicheskaya $i$ gornorudnaya promyshlennost [Metallurgical and mining industry], 2, 43-44

5. Bulat, A.F., Dyrda, V.I., Lysytsya, M.I., Grebenyuk, S.M. (2018). Numerical Simulation of the Stress-Strain State of Thin-Layer Rubber-Metal Vibration Absorber Elements Under Nonlinear Deformation, Strength of Materials, 3 (50), 387-395 